\title{
Circumferential resection margin involvement: an independent predictor of survival following surgery for oesophageal cancer
}

\author{
S P L Dexter, H Sue-Ling, M J McMahon, P Quirke, N Mapstone, I G Martin
}

\begin{abstract}
Background-For rectal carcinoma, the presence of tumour within $1 \mathrm{~mm}$ of the circumferential margin is an important independent prognostic factor for both local recurrence and survival. Similar prospective data have not been reported for oesophageal carcinoma and we wished to ascertain the prognostic importance of this variable following potentially curative resection for oesophageal carcinoma.

Aim-To prospectively assess the impact of circumferential margin involvement (tumour within $1 \mathrm{~mm}$ ) following potentially curative resection for oesophageal carcinoma.
\end{abstract}

Patients and methods-In a prospective study, resection specimens of 135 patients treated with potentially curative oesophageal resection alone were studied for the presence of tumour within $1 \mathrm{~mm}$ of the circumferential margin (margin positive), using inked margins and cross sectional slicing of the specimen. All tumours were also staged using the 1987 UICC TNM classification. Patients were followed for a mean of 19 months, and overall and cancer specific survival analysed.

Division of Surgery, School of Medicine, University of Leeds and Department of Surgery, General Infirmary at Leeds, Leeds, UK

S P L Dexter

H Sue-Ling

M J McMahon

I G Martin

Department of Pathology, School of Medicine, University of Leeds and Department of Pathology, General Infirmary at Leeds, Leeds, UK

P Quirke

N Mapstone

Correspondence to: Professor I G Martin, University Department of Surgery, South Auckland Clinical School, Middlemore Hospital, Private Bag 93311 , Otahuhu, Auckland 6, New Zealand.

igmartin@middlemore.co.nz

Accepted for publication 5 December 2000 21 months compared with 39 months in the CRM- group ( $p=0.015)$. The impact of CRM status on survival was only seen in patients with a low nodal metastatic burden ( $<25 \%$ nodes positive). The odds ratio for the risk of dying from oesophageal cancer was 2.08 when the CRM was involved $(p=0.013)$.

Conclusions-The presence of tumour within $1 \mathrm{~mm}$ of the circumferential margin following potentially curative resection for oesophageal carcinoma is an important independent prognostic variable and should be reported routinely. (Gut 2001;48:667-670)

Keywords: oesophageal cancer; survival; circumferential resection margin

Oesophageal carcinoma is the seventh most common cause of cancer deaths in the developed world and oesophageal adenocarcinoma is increasing in incidence faster than any other cancer. ${ }^{1}$ Despite recent developments in non-surgical treatment, ${ }^{2}$ surgery remains the mainstay of potentially curative treatment. The outcome following surgical resection for oesophageal cancer is generally poor but reported results vary widely between different centres. Following surgical resection, local tumour recurrence is common ${ }^{3}$ and is almost invariably followed by the death of the patient shortly afterwards.

The link between local recurrence and margin of normal tissue surrounding a resected cancer is well established for breast cancer. ${ }^{4}$ More recently, both local recurrence and survival after surgery for rectal cancer have been shown to be closely correlated with circumferential resection margin (CRM) involvement. ${ }^{5}$ In this prospective study, the finding of tumour within $1 \mathrm{~mm}$ of the circumferential (lateral) margin of the resection specimen of patients undergoing what would be regarded as a potentially curative resection was a highly significant predictor of both local recurrence and survival. This pathological variable significantly added to the prognostic information provided by conventional TNM staging alone.

To date, similar data are not available for patients undergoing surgical resection for oesophageal cancer, although a small retrospective study from this department suggested that, as for rectal cancer, the circumferential margin may well be important. ${ }^{6}$ The aim of this prospective study was to correlate the finding of tumour within $1 \mathrm{~mm}$ of the circumferential margin with survival after potentially curative oesophageal resection.

\section{Patients and methods}

Between 1990 and 1997, all patients within one hospital trust (United Leeds Teaching Hospitals Trust) who underwent a potentially curative oesophagectomy were entered prospectively into the study. Neoadjuvant therapy was not used in these patients. Resections were regarded as potentially curative if there was no macroscopic residual disease at operation and the proximal and distal surgical margins were free of tumour on pathological examination.

There were 135 patients who underwent potentially curative resection: 88 males and 47 females. Ninety eight $(73 \%)$ had an adenocarcinoma. Mean age was 64 years (range 29-82). circumferential resection margin.
Abbreviations used in this paper: CRM, 


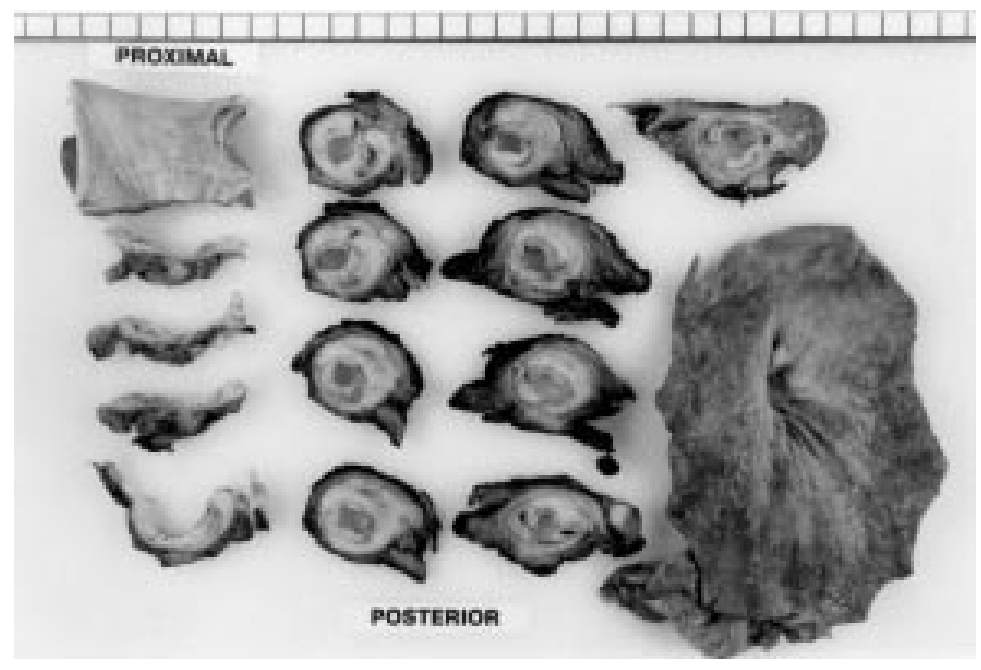

Figure 1 Oesophageal cancer resection specimen following inking of the margins and serial cross sectional sequential slicing
Table 1 Circumferential resection margin (CRM) involvement by $T$ and $N$ stage

\begin{tabular}{llll}
\hline & CRM-ve & CRM+ve & $\% C R M+v e$ \\
\hline Overall $(\mathrm{n}=135)$ & 71 & 64 & 47 \\
T1 $(\mathrm{n}=17)$ & 17 & 0 & 0 \\
T2 $(\mathrm{n}=23)$ & 19 & 4 & 17 \\
T3 $(\mathrm{n}=95)$ & 35 & 60 & 64 \\
N0 $(\mathrm{n}=55)$ & 33 & 22 & 40 \\
N1 $(\mathrm{n}=80)$ & 38 & 42 & 52
\end{tabular}

Mean node harvest per patient, 14 nodes (range 1-52).

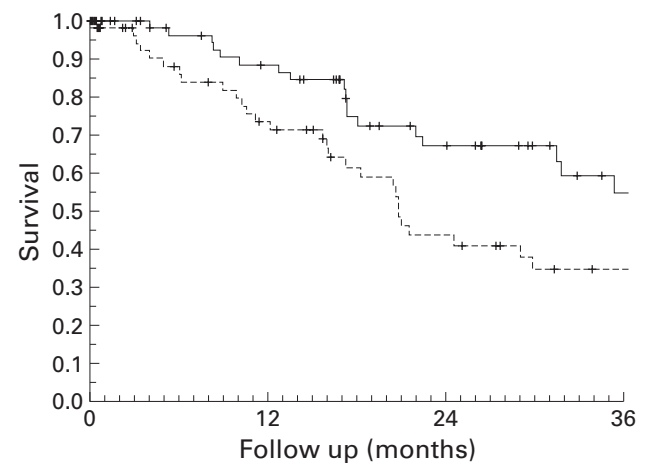

of development within the trust. While 11 consultants contributed patients to the study, the majority of resections were performed by four consultant upper gastrointestinal surgeons. In the last two years of the study almost all resections were performed by two surgeons who specialise in oesophagogastric surgery. A variety of operative techniques were used: four patients had a one stage left thoracoabdominal approach, 101 a two stage abdominal and right thoracotomy approach, four patients a three stage operation, 19 patients a thoracoscopically assisted oesophagectomy (3 stage, no thoracotomy), and five patients a transhiatal approach. There were 16 in hospital postoperative deaths (11.9\%) and 30 day mortality was $11.1 \%$.

The removed oesophagus was sent unopened to the pathology department. The outside of the specimen was painted with Indian ink and fixed for a minimum of 48 hours without opening of the tumour bearing section of the specimen. The tumours were then serially sectioned transversely and specimens examined to determine the distance between the tumour and the nearest inked margin (fig 1). Where the distance was measured as $1 \mathrm{~mm}$ or less, the CRM was considered to be involved. Other aspects of TNM staging were taken from the 1987 UICC TNM classification. ${ }^{7}$

Survival data were obtained from clinical records and cross referenced with the Yorkshire Cancer Registry. After operation, all patients were followed up by the respective surgical teams. Mean follow up was 19 months (median 16) and no patient was lost to follow up. The median follow up of patients succumbing to cancer was nine months, and 26 months in those surviving at the conclusion of the study. Cancer specific and overall survival curves to three years were constructed using KaplanMeier life table analysis. The effect of tumour type (squamous carcinoma or adenocarcinoma), degree of differentiation (well, moderate, poor), $\mathrm{T}$ stage, $\mathrm{N}$ stage, and CRM involvement on survival were calculated using Cox's multivariate regression model.
Figure 2 Overall cancer specific survival for patients with (broken line) and without (solid line) tumour within $1 \mathrm{~mm}$ of the circumferential resection margin.

\section{Results}

Tumour was seen within $1 \mathrm{~mm}$ of the CRM $(\mathrm{CRM}+)$ in $64(47 \%)$ patients. Of 37 patients with squamous cancers, $18(49 \%)$ had a positive CRM compared with 46 of 98 patients (46\%) with an adenocarcinoma. In patients with middle third tumours, $54 \%$ had a positive CRM compared with $45 \%$ of distal third tumours $(p=0.4)$. Table 1 shows the breakdown of CRM involvement by $\mathrm{T}$ and $\mathrm{N}$ stage. Overall survival at three years was 36\% (95\% confidence intervals (CI) 46-26\%), with a median survival of 21 months (95\% CI 16-25 months). Cancer specific survival at three years was $45 \%$ (95\% CI $57-34 \%$ ), with a median survival of 32 months (95\% CI 18-45 months). There was no difference in survival of patients with squamous cancers (median survival 39 months) compared with patients with adenocarcinomas (median survival 32 months; $\mathrm{p}=0.7$ ). For all subsequent survival analyses, cancer specific values will be used. The subsequent analyses were performed for overall survival and did not alter the conclusions reached.

Patients in whom CRM involvement was nosis than those patients in whom tumour was margin ( $p=0.015, \log$ rank test). Median survival for CRM positive patients was 21 months (95\% CI 19-22 months) compared with 39 months (95\% CI 17-60 months) for CRM negative patients (fig 2).

When patients were stratified into groups of low and high nodal metastatic tumour burden on the basis of less or more than $25 \%$ of nodes involved, the effect of involved circumferential margins on survival became clearer. In patients with less than $25 \%$ of positive nodes, the effect of CRM involvement was marked, with three year survival being $78 \%$ and $44 \%$, respectively $(\mathrm{p}<0.01)$ (fig 3$)$. This difference was not seen demonstrated had a significantly worse progmore than $1 \mathrm{~mm}$ from the circumferential 


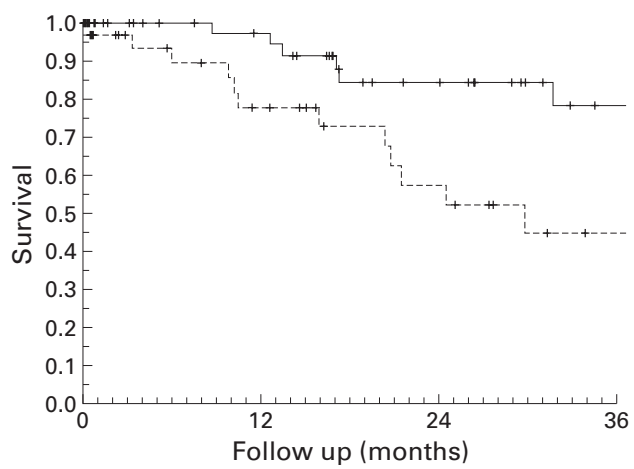

Figure 3 Survival for patients with less than 25\% of involved nodes, with (broken line) and without (solid line) tumour within $1 \mathrm{~mm}$ of the circumferential resection margin

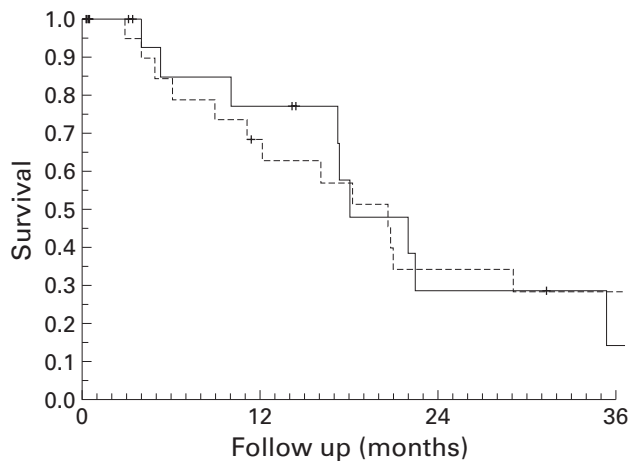

Figure 4 Survival for patients with more than $25 \%$ of involved nodes, with (broken line) and without (solid line) tumour within $1 \mathrm{~mm}$ of the circumferential resection margin

Table 2 Results of multivariate analysis

\begin{tabular}{lllll}
\hline & Beta & $S E$ & $p$ Value & $R R$ \\
\hline CRM status & 0.735 & 0.294 & 0.013 & 2.08 \\
Node status & 0.758 & 0.325 & 0.02 & 2.13
\end{tabular}

CRM, circumferential resection margin; SE, standard error; $R R$, relative risk.

in patients with more than $25 \%$ positive nodes whose survival was very poor, regardless of CRM status (median survival 21 months) (fig 4).

Using Cox's proportional hazards model, node status and CRM involvement were found to be the only two independent variables with an effect on survival (table 2).

\section{Discussion}

The results of our study confirm the importance of circumferential margin involvement in assessing prognosis after resection for oesophageal cancer. Put simply, the finding of tumour within $1 \mathrm{~mm}$ of the circumferential margin following what would otherwise be regarded as a potentially curative resection doubles the risk of the patient dying from their cancer in the short to medium term. We have shown that CRM status is an independent predictor of survival. Our multivariate analysis examined the most commonly reported pathological variables but did not investigate other prognostic factors such as vascular invasion. A larger study is required to fully assess the entire prognostic picture and indeed we are continuing to recruit to this study. One of the future aims is to investigate whether formal introduction of a more radical en bloc resection can lower the incidence of CRM involvement. It is only with such en bloc resections that the prognostic influence of other factors such as pleural invasion could also be fully assessed.

Although the study did not specifically examine patterns of recurrence, locoregional recurrence is common following oesophageal resection, with $40-50 \%$ of recurrences having a locoregional element. ${ }^{3}$ These recurrences take place early after operation (median 10 months) and result in rapid death of the patient in almost all cases (median survival of four months). ${ }^{3}$ Although median follow up of patients in our study was relatively short, the median follow up of patients not succumbing to their cancer was in excess of two years. As a consequence of the short time period usually seen between surgery and local recurrence and death, we believe that follow up of patients in our study allows us to draw valid conclusions.

The relationship between survival and CRM involvement is not simple and assumes different importance according to the extent of lymph node involvement; the benefit of clear surgical margins appears to be confined to patients with a low metastatic tumour burden. The importance of nodal tumour burden is well recognised. For patients with squamous cell carcinoma of the oesophagus, the presence of more than four or five positive nodes has been shown to have a poor prognosis. ${ }^{89}$ Similarly, a ratio of involved to resected nodes of more than $50 \%$ is also associated with a poor prognosis. ${ }^{10}$ Previous analysis of data from our department suggested that prognosis starts to worsen when more than $25 \%$ of nodes are involved. It is likely that patients with extensive nodal involvement have systemic disease at the time of surgery and the presence of CRM involvement represents only a proportion of microscopic residual disease.

As circumferential margin clearance appears to be most important in patients who have oesophageal cancer with limited locoregional spread, the role of the surgeon in such patients should therefore be to resect the oesophagus with as wide a margin of undisturbed normal tissue as possible around it. CRM involvement from oesophageal cancer is likely to remain more common than for rectal cancer because the oesophagus is surrounded closely by vital structures which cannot be resected en bloc. Furthermore, there is no specific fascial boundary to circumferential spread of oesophageal cancer, unlike the mesorectal fascia and Denonvillier's fascia for rectal cancer.

Multiple nodal involvement has proved resistant to surgical cure even by extended lymphadenectomy but patients with low grade lymph node involvement can achieve long term survival after radical surgery. ${ }^{11}{ }^{12}$ While most authors assume the survival advantage gained by radical surgery is attributable to extended lymphatic and nodal clearance, ${ }^{12-14}$ our data suggest that the benefit may be due in part to wider circumferential margins. Whatever the rationale, the technique of radical excision usually involves en bloc oesophagectomy with 
two or three field lymphadenectomy, and the individual contributions of circumferential margins and lymphadenectomy are difficult to separate. Altorki et al compared their results from en bloc oesophagectomy with standard oesophagectomy for stage III disease. ${ }^{14}$ Their standard oesophagectomy involved taking closer circumferential margins and performing a less radical lymphadenectomy. The number of positive nodes was similar in both groups but median survival was significantly less after standard resection (median survival en bloc 27 months, standard 12 months; $\mathrm{p}<0.001$ ). For node negative tumours there was also a survival difference (three year survival, en bloc $68 \%$, standard 27\%). The authors attributed the results to differences in extent of nodal clearance and understaging after standard resection in node negative patients. Data on circumferential margin involvement were not presented but survival curves for the two operations were strikingly similar to our own curves for CRM status. It is conceivable that differences in CRM involvement from the two procedures might have contributed to the differences between groups.

Neoadjuvant treatment may provide an opportunity to reduce the incidence of circumferential margin involvement in patients with bulky disease in whom primary surgery is unlikely to achieve circumferential clearance. However, this assumption remains speculative, particularly given the conflicting evidence regarding the benefit of neoadjuvant chemoradiotherapy. ${ }^{15}{ }^{16}$ In any future study of neoadjuvant therapy it would, on the basis of the data presented in this paper, be important to accurately and prospectively record the CRM status of oesophageal resection specimens.

In conclusion, we have shown that routine assessment of the circumferential margin of oesophageal cancer specimens adds significantly to the prognostic information provided by TNM staging and as such circumferential resection margin involvement should be assessed as part of the minimum pathology data set for oesophageal cancer reporting.

Funding for the study was provided by core HEFCE and NHS funding.

1 Blot WJ, Devesa SS, Kneller RW, et al. Rising incidence of adenocarcinoma of the esophagus and gastric cardia. ҰAMA 1991;265:1287-9.

2 al-Sarraf M, Martz K, Herskovic A, et al. Progress report of combined chemoradiotherapy versus radiotherapy alone in patients with esophageal cancer: an intergroup study (published erratum appears in $\mathcal{F}$ Clin Oncol 1997;15:866). F Clin lished erratum appears

3 Law SY, Fok M, Wong J. Pattern of recurrence after oesophageal resection for cancer: clinical implications. $\mathrm{Br} F$ Surg 1996;83:107-11

4 Veronesi U, Salvadori B, Luini A, et al. Breast conservation is a safe method in patients with small cancer of the breast. Long-term results of three randomised trials on 1,973 patients. Eur f Cancer 1995;31A:1574-9.

5 Adam IJ, Mohamdee MO, Martin IG, et al. Role of circumferential margin involvement in the local recurrence of rectal cancer. Lancet 1994;344:707-11.

6 Sagar PM, Johnston D, McMahon MJ, et al. Significance of circumferential resection margin involvement after oesophagectomy for cancer. Br F Surg 1993;80:1386-8.

7 Committee on TNM classification IUAC. TNM classification of malignant tumours, 4 edn. Berlin: Springer, 1987.

8 Korst RJ, Rusch VW, Venkatraman E, et al. Proposed revision of the staging classification for esophageal cancer. revision of the staging classification for eso

9 Kawahara K, Maekawa K, Okabayashi K, et al. The number Kawahara K, Maekawa K, Okabayashi K, et al. The number
of lymph node metastases influences survival in esophageal cancer. F Surg Oncol 1998;67:160-3.

10 Kelin S, Rugang Z, Dawei Z, et al. Prognostic significance of lymph node metastasis in surgical resection of oesophageal cancer. Chin Med f 1996;109:89-92.

11 Lieberman MD, Schriver CD, Bleckner S, et al. Carcinoma of the oesophagus. Prognostic significance of histological type. F Thorac Cardiovasc Surg 1994;109:130-9.

12 Akiyama H, Tsurumara M, Udagawa H. Radical lymph node dissection for cancer of the thoracic esophagus. Ann Surg 1994;220:364-73.

13 Nishimaki T, Suzuki T, Tanaka Y, et al. Evaluating the rational extent of dissection in radical esophagectomy for invasive carcinoma of the thoracic esophagus. Surg Today 1997;27:3-8.

14 Altorki NK, Girardi L, Skinner DB. En bloc esophagectomy improves survival for stage III esophageal cancer. F Thorac improves survival for stage III esopha

15 Walsh TN, Noonan N, Hollywood D, et al. A comparison of multimodal therapy and surgery for esophageal adenocarcinoma. N Engl f Med 1996;335:462-7.

16 Bosset JF, Gignoux M, Triboulet JP, et al. Chemoradiotherapy followed by surgery compared with surgery alone in squamous-cell cancer of the esophagus. $N$ Engl f Med 1997;337:161-7. 